\title{
Genome-Wide Association Study Identified Novel Genomic Loci Controlling Internode Lengths and Plant Height in Common Wheat under Different Nitrogen Treatments
}

Piyi Xing ( $\square$ pyxing@sdau.edu.cn )

Shandong Agricultural University https://orcid.org/0000-0002-7013-7438

\section{Xia Zhang}

Shandong Agricultural University

Dandan Li

Shandong Agricultural University

Honggang Wang

Shandong Agricultural University

Yinguang Bao

Shandong Agricultural University

Xingfeng Li

Shandong Agricultural University

\section{Research Article}

Keywords: Wheat, GWAS, Wheat 90K SNP assay, Plant height-related traits, Low nitrogen stress

Posted Date: October 14th, 2021

DOI: https://doi.org/10.21203/rs.3.rs-901017/v1

License: () (1) This work is licensed under a Creative Commons Attribution 4.0 International License. Read Full License 


\section{Abstract}

Nitrogen is an important nutrient for crop growth and development. Plant height-related traits can be affected by nitrogen supplementation. In this study, we performed a genome-wide association study (GWAS) on plant height, spike length, length of different internodes, and lodging resistance strength at the grain-filling stage based on a natural wheat population subjected to low nitrogen and normal (CK) treatments. GWAS analysis showed that a total of 86 quantitative trait locus (QTLs) were detected, including 13 QTLs for plant height, 10 QTLs for spike length, 19 QTLs for the length of the first internode from the top of the plant, 6 QTLs for the second internode length, 11 QTLs for the third internode length, 13 QTLs for the fourth internode length, and 14 QTLs for the fifth internode length. Compared to the CK treatment, the plant height, spike length, and fourth and fifth internode lengths were significantly affected by the low nitrogen treatment. A total of 18 QTLs responding to low nitrogen stress were detected, including three QTLs for the fourth internode length detected on 3A, 6A, and 6D chromosomes, eleven QTLs for the fifth internode length on 1A, 1B, 1D, 2A, 2B, 3A, 3B, 4A, 5B and 7B chromosomes, one QTL for spike length on $3 A$ chromosome, and one QTL for plant height on 5B chromosome. These QTLs will enhance our understanding of the genetic basis of plant height responses to nitrogen deficiency and will benefit genetic reactions to nitrogen fertilization.

\section{Introduction}

Nitrogen is an important nutrient for crop growth and development. Large quantities of nitrogen fertilizer are commonly used to obtain high yields for many crops (Cormier et al. 2016; Peng et al. 2010). In addition, modern breeding programs have mostly aimed at obtaining high yields under a high nitrogen supply (Liu et al. 2021). The high input of nitrogen fertilizers has resulted in low nitrogen use efficiency and has caused a series of environmental problems, such as soil acidification and water eutrophication (Shi et al. 2010). To solve these kinds of problems and to improve nitrogen use efficiency, the breeding of new cultivars with improved productivity in low nitrogen environments provides an effective approach to increase nitrogen use efficiency (Marino et al. 2011; Xu et al. 2014).

Plant height is an important agronomic trait in wheat, as it is a key parameter affecting the lodging and grain yield of wheat. A reduction in crop height can be associated with reduced grain yield (Law et al. 1978), and the aim of breeding programs is therefore the identification of variants with reduced height without adversely affecting yield (Wurschum et al. 2015). In general, along with a lower incidence rate of lodging, an appropriate grain number per spike, and an improved harvest index, an appropriate plant height could increase grain yield and quality (Hedden 2003).

Classical genetic studies have indicated that the plant height of bread wheat is a complex trait and that genes located on almost all 21 chromosomes can control plant height (Law et al. 1978; Snape et al. 1977). Most dwarfing genes have now been well characterized. Rht-B1 and Rht-D1, which have been successfully used in wheat breeding programs worldwide, are located on the short arms of chromosomes $4 \mathrm{~B}$ and 4D, respectively (Borner et al. 2002; Huang et al. 2003). Rht13 on chromosome 7BS has been verified to produce a marked reduction in height (Ellis et al. 2005). In addition, the photoperiod-insensitive alleles of the major photoperiod regulator $P p d-1$ have also been reported to exert pleiotropic effects on plant height in wheat (Borner et al. 1993).

Previous studies showed that different nitrogen levels had significant effects on plant height in wheat (Hussain et al. 2006; Yu et al. 2020). With increasing nitrogen levels, the plant height will increase accordingly. Plant height in wheat includes the spike length plus all internode lengths above the ground. The nitrogen response of the components of plant height in wheat is probably different. Understanding which components of plant height have significant responses to different nitrogen levels would be beneficial for wheat breeders to efficiently select related traits.

Quantitative trait loci (QTL) mapping based on linkage analysis is often used to analyze the molecular basis of complex traits. The objectives of previous studies were to map QTLs for agronomic and yield traits, uptake, and utilization efficiency of nitrogen in a recombinant inbred line (RIL) population under different supplemental nitrogen environments (An et al. 2006; Xu et al. 2014; Cui et al. 2016; Zhang et al. 2019; Sun et al. 2012). However, QTLs have rarely been reported from natural populations in environments with different levels of nitrogen supplementation.

With the rapid development of genotyping technologies and computational methods, genome-wide association studies (GWASs) are now becoming a powerful tool for detecting underlying complex natural variation traits in crops. Recently, important QTLs for agronomic traits have been found in crops based on GWAS methods (Lou et al. 2020; Pang et al. 2020; Sansaloni et al. 2020; Zhou et al. 2015; Hu et al. 2016). Furthermore, GWAS has more advantages than RIL population mapping, as it provides higher resolution and allows more variation to be detected without the need to construct populations (Huang and Han 2014). The wheat 90K SNP genotyping assay comprised approximately 90,000 gene-associated SNPs covering the whole genome (Wang et al. 2014). Compared to SSR markers, the 90K SNP assay is generally more abundant, stable, efficient, and cost-effective (Sun et al. 2017; Gao et al. 2016). In this study, we performed a GWAS using a set of 120 
winter wheat cultivars and a 90K SNP assay to identify QTLs associated with plant height-related traits under normal nitrogen environments and low nitrogen environments, with a focus on QTLs that respond to nitrogen stress.

\section{Materials And Methods}

\section{Plant Materials and Experimental design}

A total of 120 bread wheat cultivars collected from the Yellow and Huai Valley Regions of China, including Henan, Hebei, Shaanxi, and Shandong provinces, were selected as a natural population (Table S1). These cultivars were planted and harvested in 2017-2018 and 20182019. In each cropping season, all the surveyed cultivars were planted in experimental fields of Shandong Agricultural University, Tai'an city $\left(116^{\circ} 36^{\prime} \mathrm{E}, 36^{\circ} 57^{\prime} \mathrm{N}\right)$. Two treatments were applied: low nitrogen (LN) and a normal fertilized control (CK). Hereafter, "2017LN", "2017CK", "2018LN", and "2018CK" represent the treatment trials. Each block had four rows with a length of 1.5 meters spaced $25 \mathrm{~cm}$ apart.

To analyze the soil nitrogen content, samples were selected at a depth of 0-40 $\mathrm{cm}$ using a five-point sampling method in each block during the seedling stage. The nitrogen content of the soil samples was measured using the alkali-hydrolyzable nitrogen method (Fan et al. 2005). The soil nitrogen contents were the average of the samples. The soil nitrate nitrogen content in the low nitrogen environments was $90 \mathrm{mg} / \mathrm{kg}$, while that in the CK environments was $135 \mathrm{mg} / \mathrm{kg}$. Significant differences $(p<0.01)$ existed between the LN and CK blocks. Crop management was performed according to the local cultivation practices.

\section{Phenotyping}

For each cultivar surveyed, five representative tillers from different individual plants were investigated. Plant height and its component traits (Figure 1), including plant height (PH), spike length (SL), length of the first internode from the top (FIRILT), length of the second internode from the top (SECILT), length of the third internode from the top (THILT), length of the fourth internode from the top (FORILT), length of the fifth internode from the top (FIFILT), length of the sixth internode from the top (SIXILT), and the peduncle length (PL), were investigated (Figure 1). The lodging resistance strength (LRS) was measured in the field during the grain filling stage 15 days after flowering. The strength was recorded when the stem was pushed down $20 \mathrm{~cm}$ above the ground using a YYD-1 plant culm-strength meter (Hangzhou Wanshen Detection Technology Co., Ltd., HangZhou, China).

In the analysis of variance (ANOVA), the formula trait $=\sigma_{\mathrm{g}}{ }^{2}+\sigma_{\mathrm{e}}{ }^{2}+\sigma_{\mathrm{ge}}{ }^{2}+\sigma_{\mathrm{er}}{ }^{2}$ was used to calculate $\sigma_{\mathrm{g}}{ }^{2}, \sigma_{\mathrm{e}}{ }^{2}, \sigma_{\mathrm{ge}}{ }^{2}$ and $\sigma_{\mathrm{er}}{ }^{2}$. The formula $h^{2}=$ $\sigma_{\mathrm{g}}{ }^{2} /\left(\sigma_{\mathrm{g}}{ }^{2}+\sigma_{\mathrm{e}}{ }^{2}+\sigma_{\mathrm{ge}}{ }^{2} / \mathrm{n}+\sigma_{\mathrm{er}}{ }^{2} / \mathrm{nr}\right)$ was used to calculate broad-sense heritability $\left(h^{2}\right)$, where $\sigma_{\mathrm{g}}{ }^{2}, \sigma_{\mathrm{e}}{ }^{2}, \sigma_{\mathrm{ge}}{ }^{2}$, and $\sigma_{\mathrm{er}}{ }^{2}$ represent the variance components genotype, environment, genotype $x$ environment and error, respectively, $\mathrm{n}$ and $\mathrm{r}$ are the numbers of environments and replications, respectively (Lou et al. 2020; Yang et al. 2020). The calculation of pairwise correlation coefficients for these traits was conducted using the "Performance Analytics" package in R software, and the boxplot of traits was visualized using the "ggplot2" package under multiple comparisons methods and was utilized to compare the same trait in the different environments using the "LSD.test" (Fisher's least significant difference test) method. The formula $T_{\text {delt_aver }}=100 *\left(T_{C K}-T_{L N}\right) / T_{L N}$ was used to calculate the trait reaction response to $L N ; T_{C K}$ represents the trait in the CK environment, and $T_{L N}$ represents the trait in the $L N$ environment.

\section{DNA extraction and genotyping}

DNA was extracted from young leaf tissues of each cultivar using the SDS method (Cui et al. 2011). DNA quality was checked by electrophoresis on $1 \%$ agarose gels, and DNA concentration was determined with a Nanophotometer (NanoDrop Technologies Co., Ltd., Wilmington, USA). Genotyping was performed by Beijing EMTD Technology \& Investment Co., Ltd. (Beijing, China; http://www.emtd.com.cn) using the $90 \mathrm{~K}$ wheat genotyping assay (Wang et al. 2014) following the manufacturer's protocol.

The genotypic clusters for each SNP were determined using Genome Studio version 2011.1 software, filtering out SNPs with a call rate $>0.35$. After the filtering out of SNPs with a minor allele frequency of $<5 \%$ and SNPs with $>10 \%$ missing data, 120 cultivars with 20,689 SNPs were retained for further analysis. This processing was implemented in PLINK software (Purcell et al. 2007). The physical maps of all SNPs were obtained from the website (http://wheatgenomics.plantpath.ksu.edu/wpdb/assay?from=singlemessage) and are aligned to the IWGSC reference version 1.0 genome (International Wheat Genome Sequencing et al. 2018).

\section{Linkage disequilibrium and population structure analysis}

Linkage disequilibrium (LD) among markers was calculated for the A, B, and D genomes in PLINK software. The window size for linkage disequilibrium calculation was set based on the number of SNPs located in each genome. Pairwise linkage disequilibrium was measured using squared allele frequency correlations and was assessed by calculating the squared allele frequency correlation $\left(\mathrm{r}^{2}\right)$ for pairs of SNP loci (Pang et al. 2020). 
To better assess the population composition, three clustering methods were used to analyze the genetic structure of the cultivars. Admixture software was used to estimate the number of groups (K) (Alexander and Lange 2011; Sansaloni et al. 2020). Principal component analysis (PCA) was conducted using GAPIT with the default parameters to calculate principal components (PCs) (Lipka et al. 2012). A neighbor-joining tree was constructed using MEGA version X using the Kimura 2-parameter model (Kimura 1980). Bootstrap tests were performed with 1000 replications to assess statistical support for the tree topology (Felsenstein 1985). The final tree was visualized using the online website https://itol.embl.de/.

\section{Genome-wide association study}

GWAS was implemented by GAPIT packages in R software using the mixed linear model (MLM, PCA + K), which took the population structure and relative kinship into account (Zhang et al. 2010). The kinship matrix (K) was automatically calculated using the VanRaden method (VanRaden 2008). The first four principal components of the SNP data were included in the GWAS model. To combine the GWAS results from all environments, a uniform suggestive genome-wide significance threshold ( $P$ value $=1.0 \mathrm{e}-3$ ) was used. Quantile-quantile $(\mathrm{Q}-\mathrm{Q})$ plots and Manhattan plots were visualized with the R package "CMplot" (https://github.com/YinLiLin/CMplot). The R² value was used to evaluate the magnitude of the location's effects.

\section{Results}

\section{Population structure and LD analysis}

A total of 120 cultivars and 8,632 SNPs with known physical positions were retained for further analysis. The number of SNPs within 1 MB across each chromosome is shown in Figure S1. Moreover, the number of SNPs across each chromosome is shown in Table S1. These SNPs were distributed on all 21 wheat chromosomes, with 3,260, 4,310, and 1,062 SNPs in the A, B, and D subgenomes, respectively.

The population structure of the natural population was based on unlinked SNP markers (Cormier et al. 2014). According to the trend of the CV error value curve from the results of the admixture software, the $\mathrm{K}$ value may be four or five (Fig. 2a). PCA classified the population into four major groups, although there were many admixtures (Fig. 2b). The neighbor-joining tree divided the population into four major groups (Fig. $2 \mathrm{c}$ ). According to the planting area of each cultivar and the four subgroups, blue parts $(\mathrm{A})$ of the population represent most of the Shandong area cultivars registered in the 1990s, purple parts (B) of the population represent most of the Shandong area cultivars registered in the 2000s, red parts (C) of the population represent most of the Hebei area cultivars, and green parts (D) of the population represent most of the Henan area cultivars. Most of the cultivars from the same planting area clustered together, which inferred that cultivars from the same province were more closely related than those from different provinces (Fig. 2d). Overall, based on the above three methods and the planting area of each cultivar, it was appropriate to classify the association population into four subgroups.

LD was estimated by calculating the $r^{2}$ among all possible pairs of markers for each of the 21 chromosomes. The obtained $r^{2}$ values were then plotted against the physical distance for each of the three genomes separately (Figure S2) and across the whole genome. When the cutoff threshold for $r^{2}$ was defined as 0.1 , the LD decay distance for the entire genome was greater than 15 MB.

\section{Phenotypic trait analysis among subpopulations under different conditions}

We analyzed each phenotypic trait among subpopulations under different conditions. The performance of all traits in the LN and CK environments is displayed in Fig. 3. From Fig. 3, we found that the same traits of subgroups were different. By comparing the same traits under the CK and LN environments within a subgroup (Table S2), we found that the PH and PH components were both greater in the CK treatment than in the LN treatment, except for the SECILT and THILT of the C subgroup. In the ANOVA results, a significant P value of $\sigma e^{2}$ existed among the different environments (Table S3), which illustrated that the environment affected the traits. According to the comparison of all traits in the CK and LN environments, the PH, SL, FORILT, FIFILT, and LRS showed significant differences $(P<0.001)$. The results indicated that the decrease in PH in the LN treatment was mainly caused by decreases in the length of FORILT, FIFILT, and SL in the LN treatment.

Using the ANOVA results and formula of broad sense heritability, we calculated $h^{2}$ for each trait. The $h^{2}$ of the tested traits ranged from 0.668 (SIXILT) to $0.97(\mathrm{PH})$. For four traits (PH, SECILT, THILT, FORILT), $h^{2}$ was greater than 0.9 , whereas $h^{2}$ for FIRILT, LRS, PL, FIFILT, and SL was $0.884,0.875,0.862,0.810,0.799$ and 0.668 , respectively (Table S3).

The average data based on each trait were used to calculate Pearson's correlation coefficients. PH was positively correlated with SECITL, THILT, and FORILT (Figure S3); SL was negatively correlated with FORILT and FIFILT (Figure S3). PL showed a strong positive correlation with 
FIRILT (Figure S3), and the LRS showed no correlation with other traits (Figure S3), which indicated that LRS had no significant relationship with the length of internodes (Figure S3).

\section{GWAS of plant height-related traits under low nitrogen and normal environments}

To eliminate the influence of the kinship and structure of the population, we performed GWAS for all traits with the MLM method under CK and LN environments, and significant SNPs $(P<1 e-3)$, repetitive SNPs in multiple environments (all, E > 3; CK, E = 2; LN, E = 2) and SNPs located within the LD decay distance (15 M) were defined as repetitive QTLs. Manhattan plots and QQ plots for all traits are shown in Fig. 4 and Figure S4. All detected SNPS and QTLs in the CK and LN environments are displayed in Table S4. GWAS analysis showed that a total of 86 QTLs were detected in the LN and CK environments.

\section{Plant height and plant height components}

A total of 12 QTLs were detected after analysis of PH (Fig. 4; Table S4), including 7 QTLs in the CK environment and 6 QTLs in the LN environment, which were mainly distributed on chromosomes 1B, 3A, 3B, 3D, 4B, 6B, 7A, and 7B. Two QTLs, QPH.sdau-3A.1 (142 MB) and QPH.sdau-4B (555-570 MB), were detected in both the CK and LN environments, explaining 8.0-10.6\% and 9.2-10\% of the phenotypic variation, respectively (Fig. 4; Table S4, PH). Two QTLs, QPH.sdau-3B.2 (814 MB) and QPH.sdau-7A.1 (34-49 MB), were detected in the 2017CK and 2018CK environments and explained 9.5-10\% and 8.0-8.3\% of the phenotypic variation, respectively. No repetitive QTLs were detected with $\mathrm{PH}$ in the 2017LN and 2018LN environments.

A total of 10 QTLs mainly distributed on chromosomes 2A, 2B, 3A, 3B, 3D, 5A, 5B, and 7B were associated with SL, including 6 QTLs in the CK environment and 4 QTLs in the LN environment (Fig. 4; Table S4, SL). Among all QTLs, QSL.sdau-3D (602 MB) in 3D was detected in the 2017CK and 2018CK environments, while no SNP was detected in the LN environment. The phenotypic variation explained by $Q S L . s d a u-3 D$ ranged from 7.9-11\%. For FIRILT, 19 QTLs were identified on chromosomes 1A, 1B, 1D, 2A, 3A, 3B, 4B, 5A, 6A, 6B, 7A and 7B, including 14 QTLs in the CK environment and 8 QTLs in the LN environment (Fig. 4; Table S4, FIRILT). QFIRILT.sdau-3A.2 (503 MB) and QFIRILT.sdau-4B (555-570 MB) were both detected in the 2017CK, 2018CK, 2017LN, and 2018LN environments, explaining 9.8-12.8\% and 9.9-13.7\% of the phenotypic variation, respectively. QFIRILT.sdau-7B.2 (606-608 MB) was detected in the 2017CK and 2018CK environments and explained $10.9-12.3 \%$ of the phenotypic variation. A total of 6 QTLs for SECITL were identified on chromosomes 1B, 3A, 4A, 4B, 6B, and 7B, including 2 QTLs in the CK environment and 4 QTLs in the LN environment (Fig. 4; Table S4, SECITL). Among all QTLs, the QTL QSECILT.sdau-4B (535$570 \mathrm{MB}$ ) was detected in the 2017LN and 2018LN environments. The phenotypic variation of QSECILT.sdau-4B ranged from 8.6-9.9\%. For THILT, 11 QTLs were identified on chromosomes 2A, 2D, 3A, 3B, 4B, 6B, 7B and 7D, including 7 QTLs in the CK environment and 6 QTLs in the LN environment (Fig. 4; Table S4, THITL). A total of 13 QTLs for FORILT were identified on chromosomes 1B, 3A, 3B, 5B, 6B, 7A, 7B, and 7D, including 7 QTLs in the CK environment and 7 QTLs in the LN environment (Table S4, FORILT). For FIFILT, 14 QTLs were identified on chromosomes 2A, 3A, 3B, 5A, 5B, 6A, 6B, 6D, 7B and 7D, including 11 QTLs in the CK environment and 3 QTLs in the LN environment (Fig. 4; Table S4, FIFILT). From SECITL to FIFILT, no repetitive QTLs were detected in the CK or LN environments. Plant height in wheat is the spike length plus the length of all internodes above the ground, while SIXILT existed in a small number of cultivars; therefore, so it was not suitable to perform GWAS with SIXILT in the present study.

\section{Plant height-related traits}

Although PL and LRS are not components of PH, they are associated with plant morphology, which is closely related to PH. A total of $13 \mathrm{QTLS}$ were detected with PL on chromosomes $2 \mathrm{~A}, 3 \mathrm{~A}, 3 \mathrm{~B}, 4 \mathrm{~A}, 4 \mathrm{~B}, 5 \mathrm{~B}, 6 \mathrm{~A}$ and 7B, including $7 \mathrm{QTLs}$ in the CK environment and 9 QTLs in the LN environment (Fig. 4; Table S4, PL); however, none of the QTLs were repetitively deactivated. A total of 7 QTLs for LRS were detected on chromosomes 2B, 3A, 3B, 4A, and 6A, including 5 QTLs in the CK environment and 3 QTLs in the LN environment (Fig. 4; Table S4, LRS). Among them, QLRS.sdau-4A (630 MB) was detected in the 2017CK, 2018CK, 2017LN, and 2018LN environments, explaining 11.2-12.5\% of the phenotypic variation. QLRS.sdau-2B (42 MB) and QLRS.sdau-3B (657-664) were detected in the 2017LN and 2018LN environments, while QLRS.sdau-3A.1 (487 MB), QLRS.sdau-3A.3 (513 MB) and QLRS.sdau-6A (614 MB) were detected in the 2017CK and 2018CK environments.

QLRS.sdau-2B and QLRS.sdau-3B explained 12.1-12.5\% and 12.1-16.8\% of the phenotypic variation, respectively (Table S4, LRS). QLRS.sdau-3A.1, QLRS.sdau-3A.3 and QLRS.sdau-6A explained phenotypic variations of 10.8-11.4\%, 11.0-11.3\%, and 11.5-12.1\%, respectively (Table S4, LRS).

\section{GWAS of plant height-related traits in response to nitrogen stress}


According to the comparison of the same traits in the CK and LN environments, different nitrogen contents in the soil caused the difference in FORILT, FIVILT, SL, PH, and LRS. Tdelt_aver was measured as the trait that responded to nitrogen stress. Based on GWAS and T delt_aver, QTLs that responded to nitrogen stress were detected (Fig. 5; Table 1). A total of 18 QTLs that responded to nitrogen stress were detected.

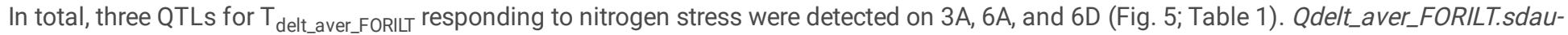
$3 A(657 \mathrm{MB})$, Qdelt_aver_FORILT.sdau-6A (18 MB) and Qdelt_aver_FORILT.sdau-6D(17 MB) explained 12.1-12.5\% and 12.1-16.8\% of the

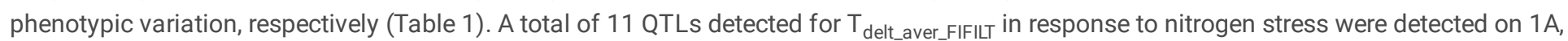
1B, 1D, 2A, 2B, 3A, 3B, 4A, 5B and 7B (Fig. 5; Table 1). Among them, Qdelt_aver_FIFILT.sdau-3A.2 (648-658 MB) had the lowest P-value and explained $36.3 \%$ of the phenotypic variation, which was considered a main-effect QTL (Table 1). Qdelt_aver_SL.sdau-3A (705 MB) was detected for $T_{\text {delt_aver_SL }}$ in response to nitrogen stress, which explained 11.3\% of the phenotypic variation (Fig. 5; Table 1). Qdelt_aver_PH-5B (701 MB) was detected for $\mathrm{T}_{\text {delt_aver_PH }}$ in response to nitrogen stress, which explained 9.5\% of the phenotypic variation (Fig. 5; Table 1). Two QTLs, Qdelt_aver_LRS.sdau-3B (150 MB) and Qdelt_aver_LRS.sdau-5B (520 MB), for T delt_aver_LRS responded to nitrogen stress and explained $12.0 \%$ and $10.4 \%$ of the phenotypic variation, respectively (Fig. 5; Table 1 ).

\section{Discussion}

\section{QTLs for plant height}

Previous genetic studies have identified numerous major QTLs for PH among 21 wheat chromosomes (Yu et al. 2020). In this study, many QTLs were also detected for PH. The GA-responsive dwarfing gene Rht-1b (Wilhelm et al. 2013; Li et al. 2013) on the short arm of chromosome $4 \mathrm{~B}$ was not detected, possibly because the materials used in this study are mainly widely used cultivars that possess the $R h t-1 b$ genes. The FIRILT, SECILT, and THILT internodes may have an association with maturity in reproductive growth. The QTLs QTHILT.sdau-4B, QSECILT.sdau-4B, and QFIRILT.sdau-4B, which could be represented by SNP BobWhite_c5694_1201 located on 4B, were detected for PH, FIRILT, SECILT, and THILT, which could be the same loci reported in a previous study on maturity (Zou et al. 2017).

\section{QTLs for the development of different internodes}

The development period of wheat $\mathrm{PH}$ is dynamic. Stem elongation occurs in an ordered sequence. When the lower internode elongates to half of its final length, the one above it begins to elongate, then the next, and so on.

In the present study, QTLs were analyzed for PH and the lengths of each internode. When the lowermost internode of wheat (FIFILT) was elongating, the QTLs that were associated with FIFILT were probably activated. As FIFILT reached half of its final length, FORILT began to elongate, and the QTLs that were associated with FORILT were activated. This phenomenon continued with the development of other internodes. In the present study, the lengths of the different internodes were correlated with each other. This means that a certain QTL might not only control a certain length of a particular internode but might also control other internodes. In other words, some QTLs were temporarily activated in the development of one internode, while some QTLs were consistently activated in the development of several internodes. According to the QTLs detected in Table S4, we found some QTLs indicating this phenomenon. First, QTLs QFIFILT.sdau-3A.1, QFORILT.sdau3A.1, QTHILT.sdau-3A.1, QSECILT.sdau-3A and QFIRILT.sdau-3A.1, which were represented by SNP wsnp_Ex_c13802_21639096 on 3A, controlled the development of FIFILT, FORILT, THILT, SECILT and FIRILT, respectively. Second, QTHILT.sdau-4B, QSECILT.sdau-4B and QFIRILT.sdau-4B, which were represented by SNPs BobWhite_c5694_1201 and BobWhite_c4311_148 on 4B, controlled the development of THILT, SECILT, and FIRILT, respectively, similar to QTHILT.sdau-7B.2, QSECILT.sdau-7B and QFIRILT.sdau-7B.2, which were represented by SNP RAC875_c7123_1703. QFIFILT.sdau-7D.2, QFORILT.sdau-7D.1 and QTHILT.sdau-7D.1, which were represented by SNP IAAV9104, controlled the development of FIFILT, FORILT, and THILT, respectively. Third, QFIFILT.sdau-3B.2 and QFORILT.sdau-3B.2, which were represented by SNP BS00091867_51, controlled the development of FIFILT and FORILT, respectively, similar to QFIFILT.sdau-6B and QFORILT.sdau-6B, which were represented by SNP Excalibur_c2737_197. Furthermore, QFORILT.sdau-3A.1 and QTHILT.sdau-3A.1, which were represented by SNP wsnp_Ex_c13802_21639096, controlled the development of FORILT and THILT, respectively, similar to QTHILT.sdau-3A.2 and QFORILT.sdau3A.2, which were represented by SNP Excalibur_c81897_420. In addition, QTHILT.sdau-3B and QFORILT.sdau-3B.2, which were represented by SNP TA004228-0191; QTHILT.sdau-6B and QFORILT.sdau-6B, which were represented by SNP Excalibur_c36771_136; QFORILT.sdau-7B and QTHILT.sdau-7B.1, which were represented by SNP Kukri_c41117_824; QFORILT.sdau-7D.2 and QTHILT.sdau-7D.2, which were represented by SNP Tdurum_contig61255_86; and QTHILT.sdau-6B and QSECILT.sdau-6B, which were represented by SNP Tdurum_contig11126_182, controlled the development of THILT and SECILT, respectively. The QTLs for each internode were activated dynamically during the development of the corresponding internode. This study provides an understanding of the genetic basis of the development of internodes.

\section{QTLs responded to low nitrogen stress}


$\mathrm{PH}$ in wheat is a complex trait; its components include SL and each internode length. As shown in Figure 6, THILT, FORILT, and FIFILT accounted for approximately $30 \%$ of the PH length. Combined with the development period of wheat (White and Edwards 2008 ), the growth of these three internodes during the period of vegetative growth leads to reproductive growth. FORILT and FIFILT showed a significant difference between the CK and LN environments in our study. Combined with the different nitrogen contents in the soil, we could infer that in the period of vegetative growth to reproductive growth, internode lengths were affected by the nitrogen content to some degree. Moreover, the $\mathrm{PH}$ in the LN environment was shorter than that in the CK environment, and the LRS in the LN environment was stronger than that in the CK environment. It could be presumed that under a degree of low nitrogen stress, the PH was reduced, and the stem became stronger. As lodging has occurred frequently in recent years, an appropriate reduction in nitrogen could increase the lodging resistance.

In this study, three QTLs for FORILT in response to nitrogen stress were detected on 3A, 6A, and 6D chromosomes; 11 QTLs for FIFILT were detected on 1A, 1B, 1D, 2A, 2B, 3A, 3B, 4A, 5B and 7B chromosomes; one QTL for SL was detected on 3A; one QTL for PH was detected on 5B; and two QTLs were detected for LRS. QTLs were detected on chromosome 3A for FORILT, FIFILT and SL, while in the nearby area, QTL qPh-3A was reported to reduce SL and FIFITL, which decreased PH under low nitrogen stress (Zhang et al. 2017), probably at a similar locus in this study. Qdelt_aver_LRS.sdau-5B, which was detected for LRS, had the lowest P-value and was located at 520552412 bp on chromosome $5 B$. TraesCS5B01G334500 (UDP-glycosyltransferase) was found near this location (upstream within 5 MB) and is considered a key gene for LRS in response to low nitrogen stress. UDP-glycosyltransferase, a key enzyme that may be involved in starch synthesis, is directly limited by the carbohydrate supply and has been shown to be impacted by $\mathrm{N}$ supply (Jiang et al., 2004).

\section{Conclusion}

In this study, we performed GWAS for plant height with spike length, the length of the first internode to the five internodes from the top during harvest, and the lodging resistance strength at the seedling stage based on a natural population in a low nitrogen and CK environments. GWAS analysis showed that a number of QTLs were detected, including 13 QTLs for PH, 10 QTLs for SL, 19 QTLs for FIRILT, 6 QTLs for SECILT, 11 QTLs for THILT, 13 QTLs for FORILT, and 14 QTLs for FIFILT. Compared to the normal environment, the of PH, FORILT, FIFILT, and SL were significantly shorter and the LRS was significantly stronger under the low nitrogen environment. GWAS was performed to detect QTLs that responded to nitrogen stress. In total, three QTLs for FORILT in response to nitrogen stress were detected on 3A, 6A, and 6D, and 11 QTLs for FIFILT were detected on 1A, 1B, 1D, 2A, 2B, 3A, 3B, 4A, 5B and 7B. In addition, one QTL for SL was detected on 3A, one QTL for PH was detected on 5B, and there were two QTLs for LRS. These QTLs may greatly deepen the understanding of the genetic basis of trait responses to nitrogen stress. The present study provided an opportunity for the detection of QTLs induced by nitrogen fertilization as well as direct evidence to dissect traits that responded to nitrogen stress induced by environmental factors.

\section{Declarations}

\section{Acknowledgments}

This work was supported by the National Key Research and Development Program of China (2016YFD0102000), National Natural Science Foundation of China (31671675), and Natural Science Foundation of Shandong Province (ZR2015CM034 and ZR2016CM30)

\section{Authorship contribution statement}

Piyi Xing performed the experiments and prepared the manuscript. Xia Zhang and Dandan Li performed partial experiments. Yinguang Bao and Honggang Wang performed partial experiments and revised the manuscript. Xingfeng Li designed the experiment and prepared the manuscript. All authors reviewed and approved the manuscript.

\section{Ethics declarations}

\section{Conflict of interest}

The authors declare that they have no conflict of interest.

\section{References}

1. Alexander DH, Lange K (2011) Enhancements to the ADMIXTURE algorithm for individual ancestry estimation. BMC Bioinformatics 12:246. doi:10.1186/1471-2105-12-246

2. An D, Su J, Liu Q, Zhu Y, Tong Y, Li J, Jing R, Li B, Li Z (2006) Mapping QTLs for nitrogen uptake in relation to the early growth of wheat (Triticum aestivum L.). Plant Soil 284(1-2):73-84. doi:10.1007/s11104-006-0030-3 
3. Borner A, Schumann E, Furste A, Coster H, Leithold B, Roder S, Weber E (2002) Mapping of quantitative trait loci determining agronomic important characters in hexaploid wheat (Triticum aestivum L.). Theor Appl Genet 105(6-7):921-936. doi:10.1007/s00122-002-0994-1

4. Borner A, Worland AJ, Plaschke J, Schumann E, Law CN (1993) Pleiotropic Effects of Genes for Reduced Height (Rht) and Day-Length Insensitivity (Ppd) on Yield and its Components for Wheat Grown in Middle Europe. Plant Breeding 111(3):204-216. doi:10.1111/j.14390523.1993.tb00631.x

5. Cormier F, Foulkes J, Hirel B, Gouache D, Moënne-Loccoz Y, Le Gouis J, Ordon F (2016) Breeding for increased nitrogen-use efficiency: a review for wheat (T. aestivumL.). Plant Breeding 135(3):255-278. doi:10.1111/pbr.12371

6. Cormier F, Le Gouis J, Dubreuil P, Lafarge S, Praud S (2014) A genome-wide identification of chromosomal regions determining nitrogen use efficiency components in wheat (Triticum aestivum L.). Theor Appl Genet 127(12):2679-2693. doi:10.1007/s00122-014-2407-7

7. Cui F, Fan X, Chen M, Zhang N, Zhao C, Zhang W, Han J, Ji J, Zhao X, Yang L, Zhao Z, Tong Y, Wang T, Li J (2016) QTL detection for wheat kernel size and quality and the responses of these traits to low nitrogen stress. Theor Appl Genet 129(3):469-484. doi:10.1007/s00122-015-2641-7

8. Cui F, Li J, Ding A, Zhao C, Wang L, Wang X, Li S, Bao Y, Li X, Feng D, Kong L, Wang H (2011) Conditional QTL mapping for plant height with respect to the length of the spike and internode in two mapping populations of wheat. Theor Appl Genet 122(8):1517-1536. doi:10.1007/s00122-011-1551-6

9. Ellis MH, Rebetzke GJ, Azanza F, Richards RA, Spielmeyer W (2005) Molecular mapping of gibberellin-responsive dwarfing genes in bread wheat. Theor Appl Genet 111(3):423-430. doi:10.1007/s00122-005-2008-6

10. Fan M, Liu X, Jiang R, Zhang F, Lu S, Zeng X, Christie P (2005) Crop Yields, Internal Nutrient Efficiency, and Changes in Soil Properties in Rice-Wheat Rotations Under Non-Flooded Mulching Cultivation. Plant Soil 277(1-2):265-276. doi:10.1007/s11104-005-7459-7

11. Felsenstein J (1985) Confidence Limits on Phylogenies: An Approach Using the Bootstrap. Evolution 39(4):783-791. doi:10.1111/j.15585646.1985.tb00420.x

12. Gao F, Liu J, Yang L, Wu X, Xiao Y, Xia X, He Z (2016) Genome-wide linkage mapping of QTL for physiological traits in a Chinese wheat population using the 90K SNP array. Euphytica 209(3):789-804. doi:10.1007/s10681-016-1682-6

13. Hedden P (2003) The genes of the Green Revolution. Trends Genet 19(1):5-9. doi:10.1016/s0168-9525(02)00009-4

14. Hu Y, Huang Y, Zhang L, Zhao H, Liu H, Xing Y, Bai X, Prasad M (2016) Genome-wide association analysis reveals flowering-related genes regulating rachis length in rice. Plant Breeding 135(6):677-682. doi:10.1111/pbr.12425

15. Huang X, Han B (2014) Natural variations and genome-wide association studies in crop plants. Annu Rev Plant Biol 65:531-551. doi:10.1146/annurev-arplant-050213-035715

16. Huang XQ, Coster H, Ganal MW, Roder MS (2003) Advanced backcross QTL analysis for the identification of quantitative trait loci alleles from wild relatives of wheat (Triticum aestivum L.). Theor Appl Genet 106(8):1379-1389. doi:10.1007/s00122-002-1179-7

17. Hussain I, Khan MA, Khan EA (2006) Bread wheat varieties as influenced by different nitrogen levels. J Zhejiang Univ Sci B 7(1):70-78. doi:10.1631/jzus.2006.B0070

18. International Wheat Genome Sequencing C, investigators IRp, Appels R, Eversole K, Feuillet C, Keller B et al (2018) Shifting the limits in wheat research and breeding using a fully annotated reference genome. Science 361 (6403). doi:10.1126/science.aar7191

19. Jiang H, Dian W, Liu F, Wu P (2004) Molecular cloning and expression analysis of three genes encoding starch synthase II in rice. Planta 218:1062-1070. doi:10.1007/s00425-003-1189-y

20. Kimura M (1980) A simple method for estimating evolutionary rates of base substitutions through comparative studies of nucleotide sequences. J Mol Evol 16(2):111-120. doi:10.1007/BF01731581

21. Law CN, Snape JW, Worland AJ (1978) The genetical relationship between height and yield in wheat. Heredity 40(1):133-151. doi:10.1038/hdy.1978.13

22. Li A, Yang W, Lou X, Liu D, Sun J, Guo X, Wang J, Li Y, Zhan K, Ling HQ, Zhang A (2013) Novel natural allelic variations at the Rht-1 loci in wheat. J Integr Plant Biol 55(11):1026-1037. doi:10.1111/jipb.12103

23. Lipka AE, Tian F, Wang Q, Peiffer J, Li M, Bradbury PJ, Gore MA, Buckler ES, Zhang Z (2012) GAPIT: genome association and prediction integrated tool. Bioinformatics 28(18):2397-2399. doi:10.1093/bioinformatics/bts444

24. Liu Y, Wang H, Jiang Z, Wang W, Xu R, Wang Q, Zhang Z, Li A, Liang Y, Ou S, Liu X, Cao S, Tong H, Wang Y, Zhou F, Liao H, Hu B, Chu C (2021) Genomic basis of geographical adaptation to soil nitrogen in rice. Nature. doi:10.1038/s41586-020-03091-W

25. Lou H, Zhang R, Liu Y, Guo D, Zhai S, Chen A, Zhang Y, Xie C, You M, Peng H, Liang R, Ni Z, Sun Q, Li B (2020) Genome-wide association study of six quality-related traits in common wheat (Triticum aestivum L.) under two sowing conditions. Theor Appl Genet. doi:10.1007/s00122-020-03704-y 
26. Marino S, Tognetti R, Alvino A (2011) Effects of varying nitrogen fertilization on crop yield and grain quality of emmer grown in a typical Mediterranean environment in central Italy. Eur J Agron 34(3):172-180. doi:10.1016/j.eja.2010.10.006

27. Pang Y, Liu C, Wang D, St Amand P, Bernardo A, Li W, He F, Li L, Wang L, Yuan X, Dong L, Su Y, Zhang H, Zhao M, Liang Y, Jia H, Shen X, Lu Y, Jiang H, Wu Y, Li A, Wang H, Kong L, Bai G, Liu S (2020) High-Resolution Genome-wide Association Study Identifies Genomic Regions and Candidate Genes for Important Agronomic Traits in Wheat. Mol Plant 13(9):1311-1327. doi:10.1016/j.molp.2020.07.008

28. Peng S, Buresh RJ, Huang J, Zhong X, Zou Y, Yang J, Wang G, Liu Y, Hu R, Tang Q, Cui K, Zhang F, Dobermann A (2010) Improving nitrogen fertilization in rice by sitespecific N management. A review. Agron Sustain Dev 30(3):649-656. doi:10.1051/agro/2010002

29. Purcell S, Neale B, Todd-Brown K, Thomas L, Ferreira MA, Bender D, Maller J, Sklar P, de Bakker PI, Daly MJ, Sham PC (2007) PLINK: a tool set for whole-genome association and population-based linkage analyses. Am J Hum Genet 81(3):559-575. doi:10.1086/519795

30. Sansaloni C, Franco J, Santos B, Percival-Alwyn L, Singh S, Petroli C, Campos J, Dreher K, Payne T, Marshall D, Kilian B, Milne I, Raubach S, Shaw P, Stephen G, Carling J, Pierre CS, Burgueno J, Crosa J, Li H, Guzman C, Kehel Z, Amri A, Kilian A, Wenzl P, Uauy C, Banziger M, Caccamo M, Pixley K (2020) Diversity analysis of 80,000 wheat accessions reveals consequences and opportunities of selection footprints. Nat Commun 11(1):4572. doi:10.1038/s41467-020-18404-w

31. Shi R, Zhang Y, Chen X, Sun Q, Zhang F, Römheld V, Zou C (2010) Influence of long-term nitrogen fertilization on micronutrient density in grain of winter wheat (Triticum aestivum L.). J Cereal Sci 51(1):165-170. doi:10.1016/j.jcs.2009.11.008

32. Snape JW, Law CN, Worland AJ (1977) Whole chromosome analysis of height in wheat. Heredity 38(1):25-36. doi:10.1038/hdy.1977.4

33. Sun C, Zhang F, Yan X, Zhang X, Dong Z, Cui D, Chen F (2017) Genome-wide association study for 13 agronomic traits reveals distribution of superior alleles in bread wheat from the Yellow and Huai Valley of China. Plant Biotechnol J 15(8):953-969. doi:10.1111/pbi.12690

34. Sun J-j, Guo Y, Zhang G-Z, Gao M-g, Zhang G-h, Kong F-m, Zhao Y, Li S-s (2012) QTL mapping for seedling traits under different nitrogen forms in wheat. Euphytica 191(3):317-331. doi:10.1007/s10681-012-0834-6

35. VanRaden PM (2008) Efficient methods to compute genomic predictions. J Dairy Sci 91(11):4414-4423. doi:10.3168/jds.2007-0980

36. Wang S, Wong D, Forrest K, Allen A, Chao S, Huang BE, Maccaferri M, Salvi S, Milner SG, Cattivelli L, Mastrangelo AM, Whan A, Stephen S, Barker G, Wieseke R, Plieske J, International Wheat Genome Sequencing C, Lillemo M, Mather D, Appels R, Dolferus R, Brown-Guedira G, Korol A, Akhunova AR, Feuillet C, Salse J, Morgante M, Pozniak C, Luo MC, Dvorak J, Morell M, Dubcovsky J, Ganal M, Tuberosa R, Lawley C, Mikoulitch I, Cavanagh C, Edwards KJ, Hayden M, Akhunov E (2014) Characterization of polyploid wheat genomic diversity using a high-density 90,000 single nucleotide polymorphism array. Plant Biotechnol J 12(6):787-796. doi:10.1111/pbi.12183

37. White J, Edwards J (2008) Wheat growth and development

38. Wilhelm EP, Mackay IJ, Saville RJ, Korolev AV, Balfourier F, Greenland AJ, Boulton MI, Powell W (2013) Haplotype dictionary for the Rht-1 loci in wheat. Theor Appl Genet 126(7):1733-1747. doi:10.1007/s00122-013-2088-7

39. Wurschum T, Langer SM, Longin CF (2015) Genetic control of plant height in European winter wheat cultivars. Theor Appl Genet 128(5):865-874. doi:10.1007/s00122-015-2476-2

40. Xu Y, Wang R, Tong Y, Zhao H, Xie Q, Liu D, Zhang A, Li B, Xu H, An D (2014) Mapping QTLs for yield and nitrogen-related traits in wheat: influence of nitrogen and phosphorus fertilization on QTL expression. Theor Appl Genet 127(1):59-72. doi:10.1007/s00122-013-2201-y

41. Yang J, Zhou Y, Hu W, Zhang Y, Zhou Y, Chen Y, Wang X, Zhao H, Cao T, Liu Z (2020) Unlocking the relationships among population structure, plant architecture, growing season, and environmental adaptation in Henan wheat cultivars. BMC Plant Biol 20(1):469. doi:10.1186/s12870-020-02674-z

42. Yu M, Liu ZH, Yang B, Chen H, Zhang H, Hou DB (2020) The contribution of photosynthesis traits and plant height components to plant height in wheat at the individual quantitative trait locus level. Sci Rep 10(1):12261. doi:10.1038/s41598-020-69138-0

43. Zhang N, Fan X, Cui F, Zhao C, Zhang W, Zhao X, Yang L, Pan R, Chen M, Han J, Ji J, Liu D, Zhao Z, Tong Y, Zhang A, Wang T, Li J (2017) Characterization of the temporal and spatial expression of wheat (Triticum aestivum L.) plant height at the QTL level and their influence on yield-related traits. Theor Appl Genet 130:1235-1252. doi:10.1007/s00122-017-2884-6

44. Zhang M, Gao M, Zheng H, Yuan Y, Zhou X, Guo Y, Zhang G, Zhao Y, Kong F, An Y, Li S (2019) QTL mapping for nitrogen use efficiency and agronomic traits at the seedling and maturity stages in wheat. Mol Breeding 39 (5). doi:10.1007/s11032-019-0965-8

45. Zhang Z, Ersoz E, Lai CQ, Todhunter RJ, Tiwari HK, Gore MA, Bradbury PJ, Yu J, Arnett DK, Ordovas JM, Buckler ES (2010) Mixed linear model approach adapted for genome-wide association studies. Nat Genet 42(4):355-360. doi:10.1038/ng.546

46. Zhou Z, Jiang Y, Wang Z, Gou Z, Lyu J, Li W, Yu Y, Shu L, Zhao Y, Ma Y, Fang C, Shen Y, Liu T, Li C, Li Q, Wu M, Wang M, Wu Y, Dong Y, Wan W, Wang X, Ding Z, Gao Y, Xiang H, Zhu B, Lee SH, Wang W, Tian Z (2015) Resequencing 302 wild and cultivated accessions identifies genes related to domestication and improvement in soybean. Nat Biotechnol 33(4):408-414. doi:10.1038/nbt.3096

47. Zou J, Semagn K, Iqbal M, N'Diaye A, Chen H, Asif M, Navabi A, Perez-Lara E, Pozniak C, Yang R-C, Randhawa H, Spaner D (2017) Mapping QTLs Controlling Agronomic Traits in the 'Attila' × 'CDC Go' Spring Wheat Population under Organic Management using 90K

Page 9/17 
SNP Array. Crop Sci 57(1):365-377. doi:10.2135/cropsci2016.06.0459

\section{Tables}

Table 1 All QTLs responding to nitrogen stress were associated with PH-related traits, including R2 and P-value 


\begin{tabular}{|c|c|c|c|c|c|c|c|}
\hline Trait & QTL & SNP & Chromosome & Position & P.value & effect & $\mathrm{R} 2$ \\
\hline \multirow[t]{3}{*}{ delt_aver_FORILT } & $\begin{array}{l}\text { Qdelt_aver_FORILT.sdau- } \\
\text { 3A }\end{array}$ & RAC875_c38647_290 & $3 \mathrm{~A}$ & 657945961 & $\begin{array}{l}9.08 \mathrm{E}- \\
04\end{array}$ & -0.094 & 0.103 \\
\hline & $\begin{array}{l}\text { Qdelt_aver_FORILT.sdau- } \\
6 \mathrm{~A}\end{array}$ & Excalibur_c49745_628 & $6 \mathrm{~A}$ & 18464438 & $\begin{array}{l}3.32 \mathrm{E}- \\
04\end{array}$ & 0.117 & 0.121 \\
\hline & $\begin{array}{l}\text { Qdelt_aver_FORILT.sdau- } \\
\text { 6D }\end{array}$ & Ra_c29107_289 & $6 \mathrm{D}$ & 17503572 & $\begin{array}{l}8.02 \mathrm{E}- \\
04\end{array}$ & -0.094 & 0.105 \\
\hline \multirow[t]{19}{*}{ delt_aver_FIVILT } & $\begin{array}{l}\text { Qdelt_aver_FIVILT.sdau- } \\
1 \mathrm{~A}\end{array}$ & Excalibur_c58154_84 & $1 \mathrm{~A}$ & 12314552 & $\begin{array}{l}8.97 \mathrm{E}- \\
04\end{array}$ & -0.602 & 0.106 \\
\hline & $\begin{array}{l}\text { Qdelt_aver_FIVILT.sdau- } \\
\text { 1B }\end{array}$ & Tdurum_contig30712_430 & $1 \mathrm{~B}$ & 111368423 & $\begin{array}{l}4.70 \mathrm{E}- \\
05\end{array}$ & 1.477 & 0.164 \\
\hline & $\begin{array}{l}\text { Qdelt_aver_FIVILT.sdau- } \\
\text { 1D }\end{array}$ & wsnp_Ex_c64327_63176640 & $1 \mathrm{D}$ & 19267156 & $\begin{array}{l}5.39 E- \\
04\end{array}$ & -0.793 & 0.116 \\
\hline & \multirow[t]{6}{*}{$\begin{array}{l}\text { Qdelt_aver_FIVILT.sdau- } \\
\text { 2A }\end{array}$} & Excalibur_c81078_578 & $2 \mathrm{~A}$ & 178472510 & $\begin{array}{l}1.29 \mathrm{E}- \\
05\end{array}$ & -2.144 & 0.191 \\
\hline & & Ex_c23985_1460 & $2 \mathrm{~A}$ & 182146095 & $\begin{array}{l}1.29 \mathrm{E}- \\
05\end{array}$ & -2.144 & 0.191 \\
\hline & & BobWhite_rep_c49432_162 & $2 \mathrm{~A}$ & 185122040 & $\begin{array}{l}1.29 \mathrm{E}- \\
05\end{array}$ & -2.144 & 0.191 \\
\hline & & Excalibur_c42815_713 & $2 \mathrm{~A}$ & 195819274 & $\begin{array}{l}9.58 E- \\
04\end{array}$ & 0.843 & 0.105 \\
\hline & & Kukri_c17989_527 & $2 \mathrm{~A}$ & 195819414 & $\begin{array}{l}9.58 \mathrm{E}- \\
04\end{array}$ & -0.843 & 0.105 \\
\hline & & wsnp_Ex_c32079_40793255 & $2 \mathrm{~A}$ & 196521584 & $\begin{array}{l}9.58 \mathrm{E}- \\
04\end{array}$ & 0.843 & 0.105 \\
\hline & \multirow[t]{2}{*}{$\begin{array}{l}\text { Qdelt_aver_FIVILT.sdau- } \\
\text { 2B }\end{array}$} & TA005635-0459 & $2 \mathrm{~B}$ & 161406179 & $\begin{array}{l}1.54 \mathrm{E}- \\
04\end{array}$ & -1.202 & 0.140 \\
\hline & & Excalibur_c23723_141 & $2 \mathrm{~B}$ & 161409259 & $\begin{array}{l}8.65 \mathrm{E}- \\
04\end{array}$ & -0.971 & 0.107 \\
\hline & $\begin{array}{l}\text { Qdelt_aver_FIVILT.sdau- } \\
\text { 3A.1 }\end{array}$ & Kukri_c41361_186 & $3 \mathrm{~A}$ & 432734 & $\begin{array}{l}3.95 \mathrm{E}- \\
04\end{array}$ & 0.692 & 0.122 \\
\hline & \multirow[t]{3}{*}{$\begin{array}{l}\text { Qdelt_aver_FIVILT.sdau- } \\
\text { 3A.2 }\end{array}$} & Excalibur_c46600_919 & $3 \mathrm{~A}$ & 648027721 & $\begin{array}{l}6.28 \mathrm{E}- \\
09\end{array}$ & -2.997 & 0.363 \\
\hline & & Excalibur_c1001_586 & $3 A$ & 648260065 & $\begin{array}{l}6.28 \mathrm{E}- \\
09\end{array}$ & -2.997 & 0.363 \\
\hline & & BobWhite_c17879_519 & $3 A$ & 658905380 & $\begin{array}{l}6.28 \mathrm{E}- \\
09\end{array}$ & -2.997 & 0.363 \\
\hline & $\begin{array}{l}\text { Qdelt_aver_FIVILT.sdau- } \\
\text { 3B }\end{array}$ & GENE-1167_104 & $3 B$ & 684256078 & $\begin{array}{l}8.45 \mathrm{E}- \\
04\end{array}$ & -0.713 & 0.108 \\
\hline & $\begin{array}{l}\text { Qdelt_aver_FIVILT.sdau- } \\
\text { 4A }\end{array}$ & Excalibur_c24511_1196 & $4 \mathrm{~A}$ & 613812680 & $\begin{array}{l}9.15 \mathrm{E}- \\
04\end{array}$ & -1.153 & 0.106 \\
\hline & $\begin{array}{l}\text { Qdelt_aver_FIVILT.sdau- } \\
5 B\end{array}$ & JD_c947_819 & $5 B$ & 72100053 & $\begin{array}{l}7.93 \mathrm{E}- \\
04\end{array}$ & -0.661 & 0.109 \\
\hline & $\begin{array}{l}\text { Qdelt_aver_FIVILT.sdau- } \\
\text { 7B }\end{array}$ & Excalibur_rep_c92684_316 & 7B & 741244806 & $\begin{array}{l}1.13 E- \\
04\end{array}$ & -1.504 & 0.147 \\
\hline delt_aver_SL & Qdelt_aver_SL.sdau-3A & Excalibur_c24354_465 & $3 \mathrm{~A}$ & 705309554 & $\begin{array}{l}5.43 E- \\
04\end{array}$ & -0.118 & 0.113 \\
\hline delt_aver_PH & Qdelt_aver_PH-5B & Excalibur_c2618_1723 & $5 B$ & 701155513 & $\begin{array}{l}6.95 \mathrm{E}- \\
04\end{array}$ & -0.046 & 0.096 \\
\hline \multirow[t]{2}{*}{ delt_aver_LRS } & Qdelt_aver_LRS.sdau-3B & Excalibur_c35491_788 & $3 B$ & 150657226 & $\begin{array}{l}3.88 \mathrm{E}- \\
04\end{array}$ & 0.532 & 0.121 \\
\hline & Qdelt_aver_LRS.sdau-5B & BS00042014_51 & $5 B$ & 520552412 & $\begin{array}{l}9.28 \mathrm{E}- \\
04\end{array}$ & 0.193 & 0.104 \\
\hline
\end{tabular}




\section{Figures}

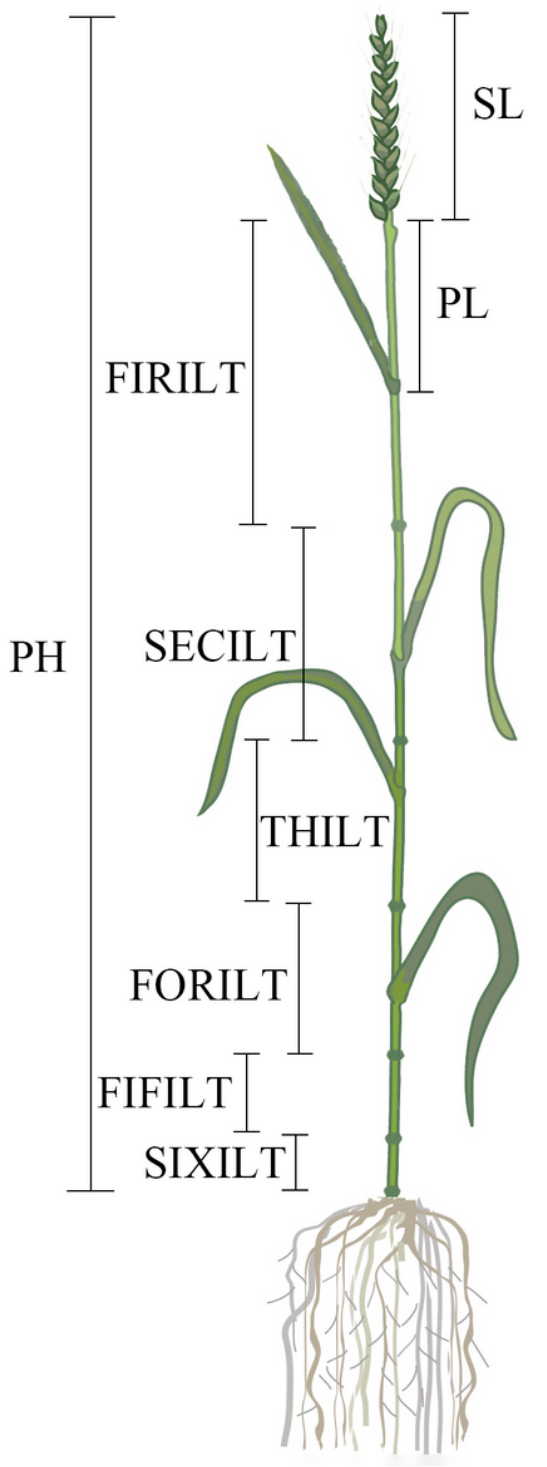

Figure 1

Schematic diagram of wheat plant morphology and $\mathrm{PH}$-related traits 


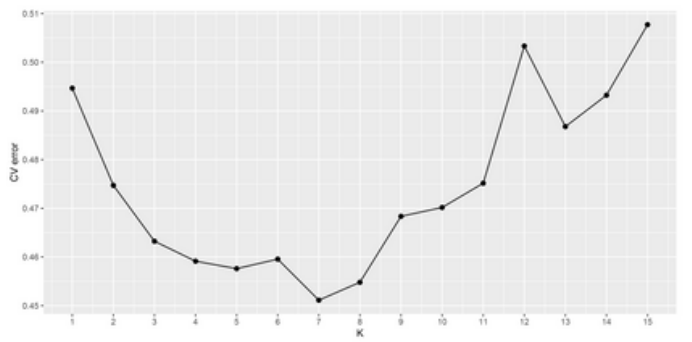

a

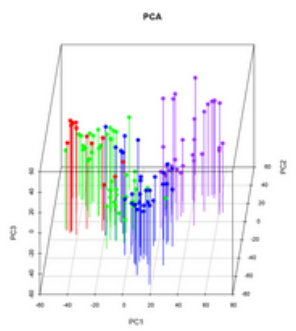

b

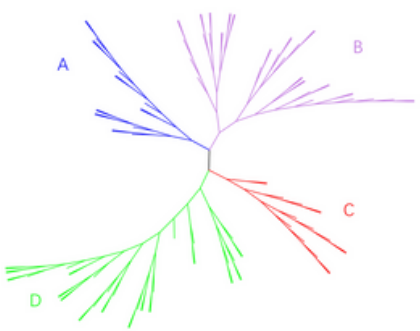

c

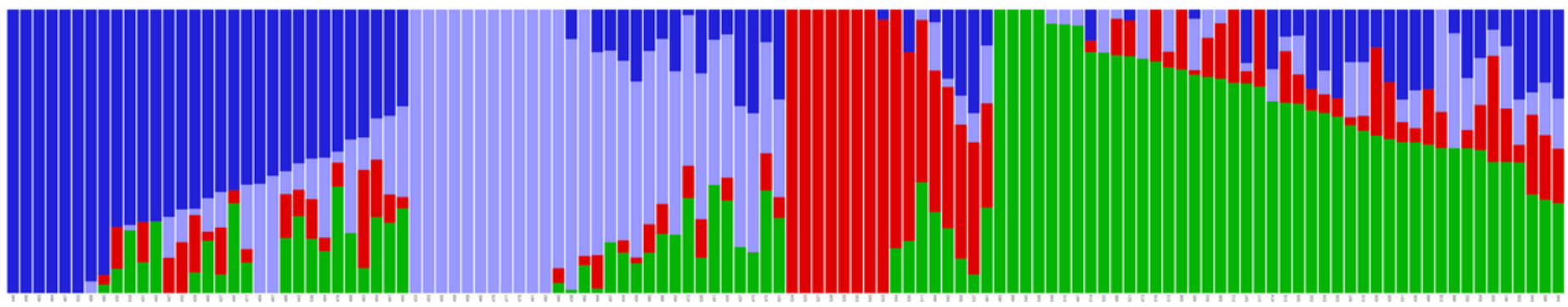

d

Figure 2

Population structure of the natural population based on 90K SNP genotyping The same colors among b, c, and d indicate the same accessions. a, Plot of CV error against putative $\mathrm{K}$ ranging from 1 to 15; b, Plot of the first principal component against the second and third principal components; c, Neighbor-joining phylogenetic tree of 120 wheat cultivars. Colors of tree branches indicate matching of the inferior population by admixture. $d$ Stacked bar plot of the ancestry relationship of 120 cultivars 

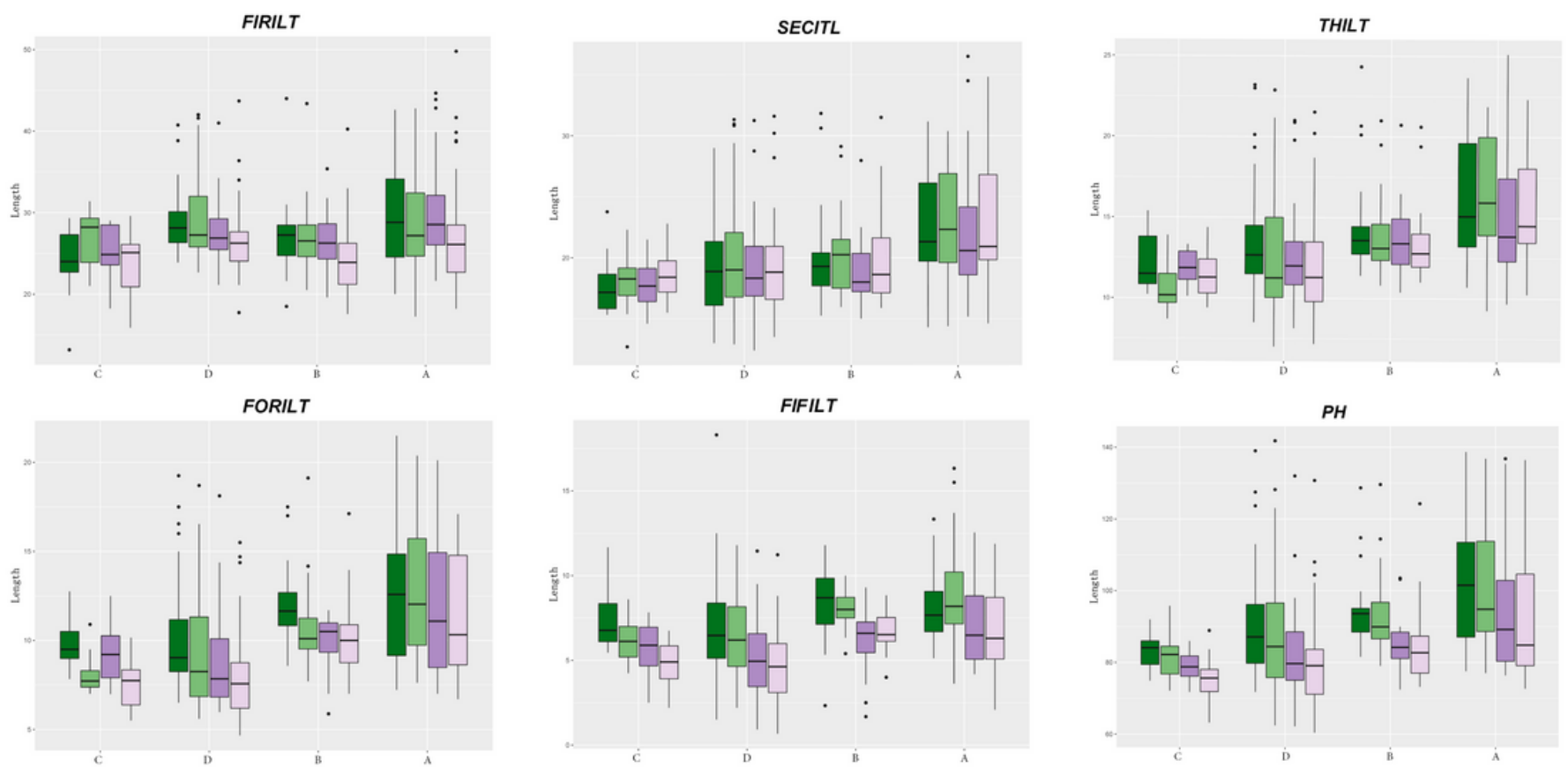

$P L$

$S L$
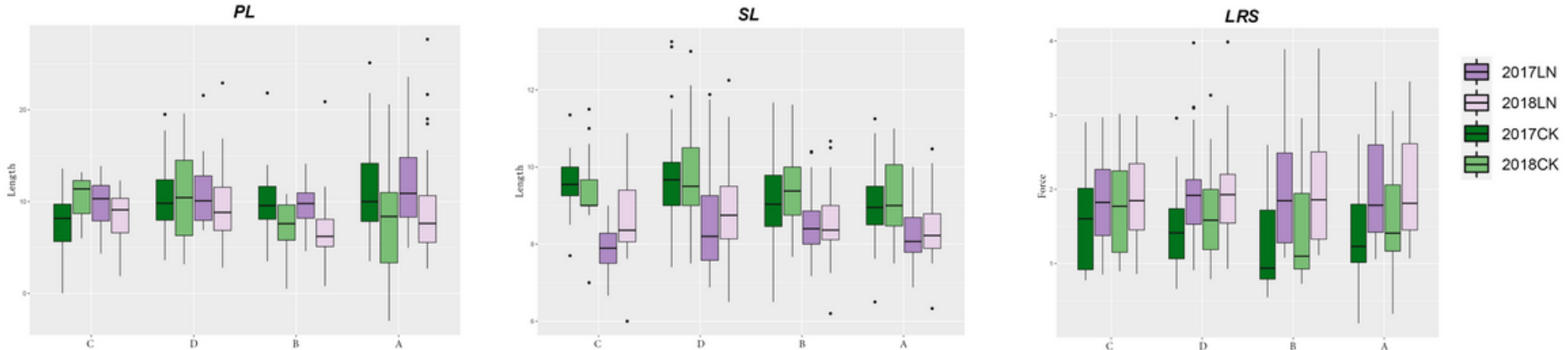

Figure 3

Comparison of the same trait in LN and CK environments and four subpopulations using boxplots 

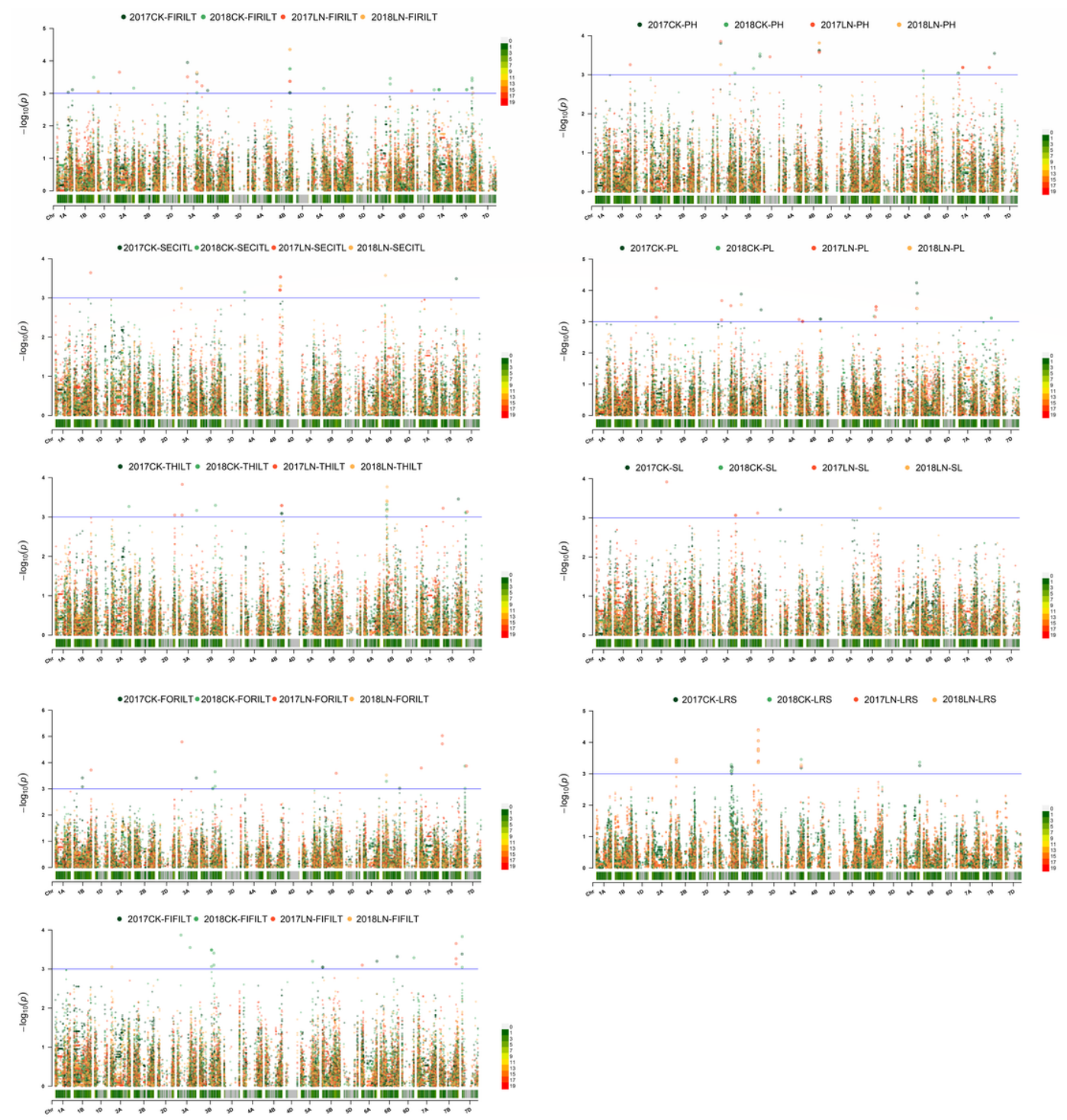

Figure 4

Manhattan plot of significant SNPs for PH-related traits 

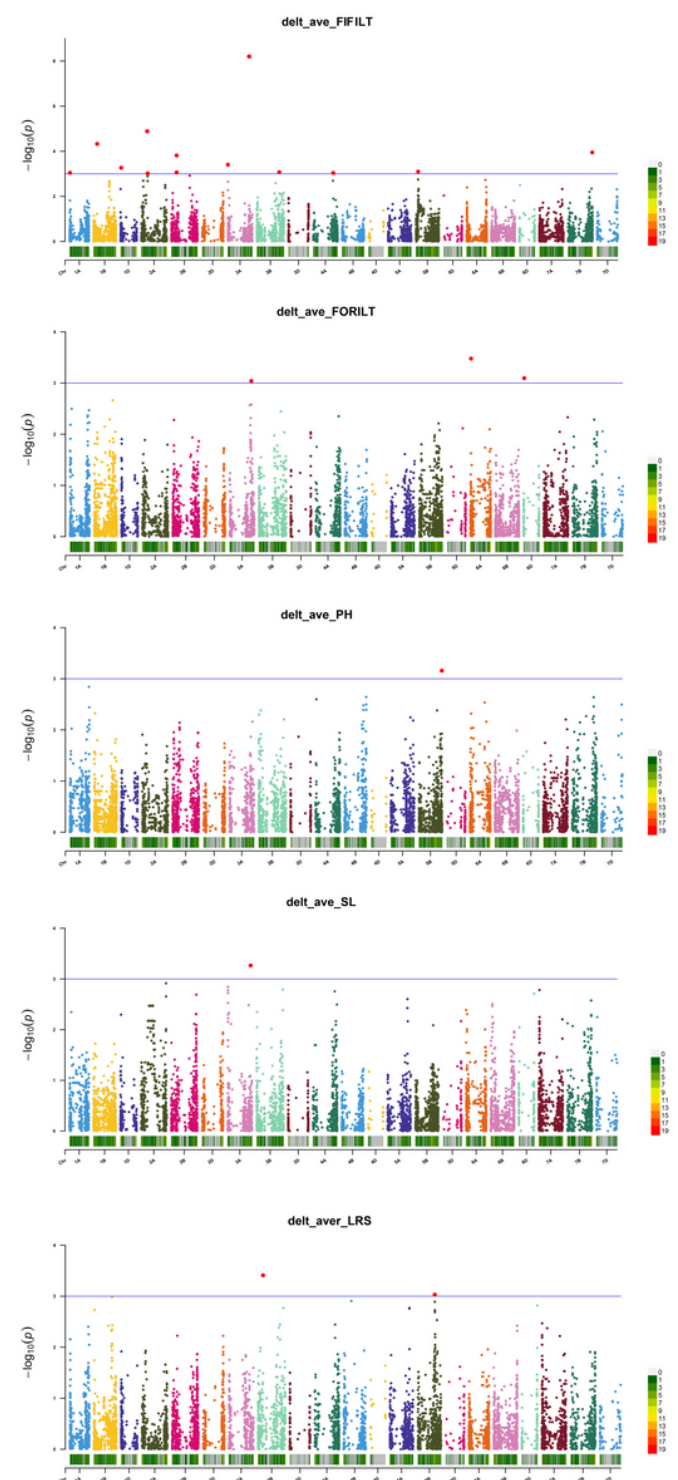

\section{Figure 5}

Manhattan plot of significant SNPs for PH-related traits under LN stress

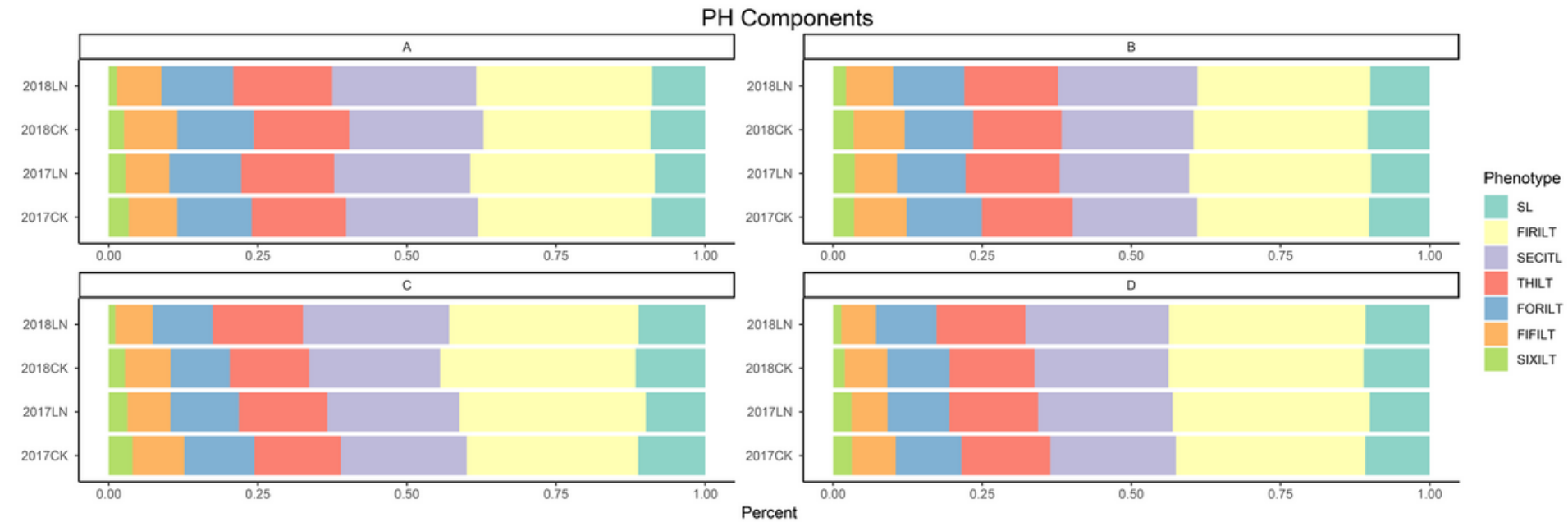

Figure 6

Percentage of $\mathrm{PH}$ components in different environments and subpopulations 


\section{Supplementary Files}

This is a list of supplementary files associated with this preprint. Click to download.

- Figures1.tif

- Figures2.tif

- Figures3.tif

- Figures4.tif

- Figures5.tif

- Tables1.xlsx

- Tables2.xlsx

- TableS3.xlsx

- TableS4.xlsx 